\title{
Self-assembled Multilayers of Silica Nanospheres for Defect Reduction in Non- and Semipolar Gallium Nitride Epitaxial Layers
}

\author{
Tongtong Zhu,,$^{, \dagger}$ Tao Ding, ${ }^{\dagger, \dagger}$ Fengzai Tang, ${ }^{\dagger}$ Yisong Han, ${ }^{\dagger}$ Muhammad Ali, ${ }^{\S}$ Tom Badcock, ${ }^{\S}$
}

Menno J. Kappers, ${ }^{\dagger}$ Andrew J. Shields, ${ }^{\S}$ Stoyan K. Smoukov, ${ }^{*}{ }^{\dagger}$ and Rachel A. Oliver ${ }^{\dagger}$

${ }^{\dagger}$ Department of Materials Science and Metallurgy, University of Cambridge, 27 Charles Babbage Road, Cambridge CB3 0FS, United Kingdom

${ }^{\ddagger}$ Nanophotonics Centre, Cavendish Laboratory, University of Cambridge, Cambridge CB3 0HE, United Kingdom

${ }^{\S}$ Cambridge Research Laboratory, Toshiba Research Europe Limited, 208 Science Park, Milton Road, Cambridge CB4 0GZ, United Kingdom

\section{Supporting Information}

ABSTRACT: Non- and semipolar GaN have great potential to improve the efficiency of light emitting devices due to much reduced internal electric fields. However, heteroepitaxial GaN growth in these crystal orientations suffers from very high dislocation and stacking faults densities. Here, we report a facile method to obtain low defect density non- and semipolar heteroepitaxial GaN via selective area epitaxy using self-assembled multilayers of silica nanospheres (MSN). Nonpolar (11-20) and semipolar (11-22) GaN layers with high crystal quality have been achieved by epitaxial integration of the MSN and a simple one-step overgrowth process, by which both dislocation and basal plane stacking fault densities can be significantly reduced. The underlying defect reduction mechanisms include epitaxial growth through the MSN covered template, island nucleation via nanogaps in the MSN, and lateral overgrowth and coalescence above the MSN. InGaN/GaN multiple quantum wells structures grown on a
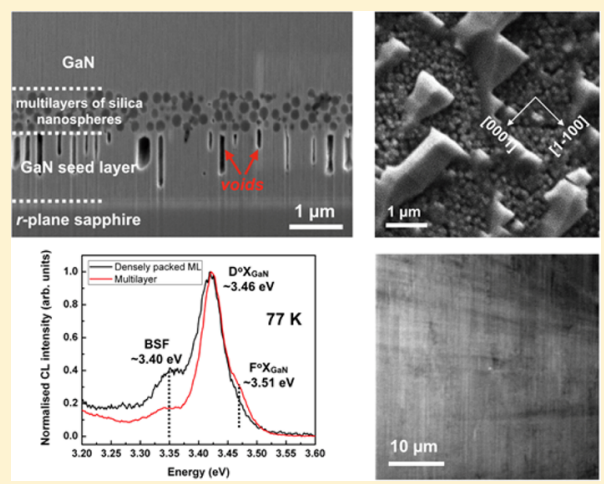
nonpolar GaN/MSN template show more than 30-fold increase in the luminescence intensity compared to a control sample without the MSN. This self-assembled MSN technique provides a new platform for epitaxial growth of nitride semiconductors and offers unique opportunities for improving the material quality of GaN grown on other orientations and foreign substrates or heteroepitaxial growth of other lattice-mismatched materials.

\section{INTRODUCTION}

GaN-based light emitting diodes (LEDs) have attracted a significant amount of attention over the past decade, owing to their high optical efficiency in the blue to UV spectral range. Most research and commercial products utilize the conventional polar c-plane orientation. However, due to the large discontinuities in the spontaneous and piezoelectric polarization at the InGaN/GaN interface, large internal electric fields along the $c$-axis separate the electron and hole wave functions and result in a lower radiative recombination efficiency and longer carrier lifetimes, and may contribute to efficiency droop in LEDs. ${ }^{1}$ Growth of non- and semipolar GaN-based structures is of interest due to the fact that it offers a crystallographic solution to reduce the internal electric fields, potentially allowing significant improvements in light emission efficiencies. Nonpolar heterostructures can also be used to produce linearly polarized light emission., ${ }^{2,3}$ Semipolar (11-22) GaN-based structures have been shown to have great potential for achieving efficient green and yellow LEDs. ${ }^{4}$

Up to now, however, research and development on non- and semipolar $\mathrm{GaN}$ is hindered by the lack of high quality $\mathrm{GaN}$ pseudosubstrates with low defect densities. Non- and semipolar bulk GaN substrates remain expensive and small in size (typically $1 \times 0.5 \mathrm{~cm}^{2}$ ), limiting their relevance to commercial consumer products. On the other hand, heteroepitaxial growth of non- and semipolar $\mathrm{GaN}$ is very challenging and results in typical densities of basal plane stacking faults (BSFs) and dislocations in excess of $10^{6} \mathrm{~cm}^{-1}$ and $10^{10} \mathrm{~cm}^{-2}$, respectively, whereas $c$-plane $\mathrm{GaN}$ templates typically have a dislocation density of $\sim 10^{8} \mathrm{~cm}^{-2}$ and no BSFs. Numerous efforts have been made to reduce the defect densities in heteroepitaxial nonand semipolar GaN. Thus far, in situ defect reduction schemes, such as the application of low-temperature GaN nucleation layers $(\mathrm{NLs})^{6}$ or AlN NLs, ${ }^{7}$ the use of silicon nitride ${ }^{8}$ or scandium nitride interlayers, ${ }^{9}$ followed by three-dimensional (3D) growth and coalescence, direct growth without a lowtemperature $\mathrm{NL},{ }^{10}$ and very high silicon doping ${ }^{11}$ can only reduce the dislocation density down to the $10^{9} \mathrm{~cm}^{-2}$ regime, while the BSF density remains well above $10^{5} \mathrm{~cm}^{-1}$. Ex situ methods, such as epitaxial lateral overgrowth (ELOG) or the

Received: November 4, 2015

Revised: December 22, 2015

Published: December 29, 2015 
use of patterned substrates, appear more promising, as they can more effectively reduce both dislocation and BSF densities. ${ }^{5,12}$ However, given the complex processing efforts involved, the fairly limited size of the low defect density areas (typically a few microns wide in nonpolar ELOG), and/or the requirement to grow very thick layers with associated wafer bowing problems, ${ }^{13}$ this may not be a very cost-effective way of achieving larger scale non- and semipolar GaN templates with very low defect densities. Recently, the emission efficiency of a-plane GaN LEDs was improved by incorporating silica nanospheres in between 3D GaN islands, ${ }^{14}$ although only a modest reduction in dislocation density was demonstrated and there was no observable impact on BSFs, possibly due to the fact that the authors did not cover the entire surface with nanospheres, thus limiting the dislocation filtering efficiency. The defect reduction mechanisms of all the methods mentioned above either rely on first bending and then annihilating the dislocations during the subsequent regrowth process or blocking the defects with an amorphous mask layer. Given that the BSFs are confined on (0001) planes and cannot be bent easily, BSFs tend to merge and exhibit an increase in the total length, so that their overall density is only slightly reduced when employing dislocation filtering methods that do not use physical masks. Therefore, reducing the BSF densities especially in nonpolar orientations remains very challenging, unless reorientation of the BSFs is enabled. ${ }^{12}$

Here we demonstrate the dramatic reduction of defects in nonpolar (11-20) GaN epilayers on $r$-plane sapphire substrates using a porous mask formed using self-assembled multilayers of silica nanospheres. During the metal-organic chemical vapor deposition (MOCVD) studies, we observed the following three phenomena: (1) the crystal epitaxy is preserved during selective area deposition through the nanogaps present in the multilayered silica nanospheres; (2) the formation of voids during the initial regrowth process and the exposure of less defective regions in the $\mathrm{GaN}$ seed layer below the nanospheres; and (3) lateral overgrowth and coalescence above the silica nanospheres. It is shown that $\mathrm{InGaN} / \mathrm{GaN}$ multiple quantum well (MQW) structures grown on MSN masked GaN templates show significantly enhanced optical performance. In addition, the MSN masking technique is also applied to the epitaxial growth of semipolar (11-22) GaN, and, here too, significant defect reduction is achieved.

\section{RESULTS AND DISCUSSION}

2.1. Nonpolar (11-20) a-plane GaN. The integration of self-assembled multilayers of silica nanospheres and the subsequent $\mathrm{GaN}$ regrowth via selective area deposition are shown schematically in Figure 1a. After an $\sim 1 \mu$ m thick GaN seed layer with a smooth surface was grown on $r$-plane sapphire by MOCVD, ${ }^{5}$ an oxygen plasma treatment was carried out for 5 min to make the GaN surface hydrophilic. Silica nanospheres $(\mathrm{d}=\sim 180 \mathrm{~nm})$ dispersed in ethanol were subsequently coated onto the $\mathrm{GaN}$ seed layer using convective assembly with a varying deposition rate $(\mathrm{mm} / \mathrm{min})$, which can result in the controlled formation of a monolayer (ML) or multilayers of silica nanospheres. ${ }^{15}$ After the silica nanosphere deposition, the samples were reintroduced into the MOCVD reactor for the $\mathrm{GaN}$ regrowth. The cross-sectional SEM image of a fully coalesced multilayer sample prepared with a focused ion beam (FIB) is shown in Figure 1b. It is seen that there are vertically elongated voids below the multilayers of nanospheres in the $\mathrm{GaN}$ seed layer, which were not present in the original seed


Figure 1. (a) Schematic illustrating the regrowth process on a GaN seed layer covered with multilayers of silica nanospheres and the dislocation reduction process (dark lines represent the dislocations), viewed along the $c$-axis [0001]. (b) Cross-sectional SEM image of a fully coalesced multilayer sample. Low temperature (15 K) (c) SEM and $\mathrm{CL}$ images of a multilayer sample stopped during the initial stages of growth, taken at (d) GaN NBE emission at $3.46 \mathrm{eV}$ and (e) BSF emission energy at $3.40 \mathrm{eV}$. White dotted circles in each image encompass an elongated island emitting at both GaN and BSF energies. Red dotted circle indicates an area of only $\mathrm{GaN}$ emission but no BSF emission originating from the GaN filling the nanogaps between the silica nanospheres.

layer growth, ${ }^{8}$ nor in the monolayer samples, and are not related to the cross-sectional sample preparation (since cleaved multilayer samples also show voids) (See Supporting Information and Figures S1), suggesting that these voids were formed during the initial regrowth process at high temperature.

In conventional selective area deposition methods, the GaN regrowth commences on the unmasked seed regions and proceeds by laterally overgrowing the masked areas, and polycrystalline nucleation on the mask does not occur. ${ }^{16,17}$ In this novel approach, the ensemble of nanospheres does not act as a conventional mask like the lithographically defined oxide stripes or patterns in an ELOG process, but rather as a porous masking material covering the entire surface. In the example of the multilayer specimen, the cross-sectional SEM image in Figure $1 \mathrm{~b}$ reveals that the MSN is not perfectly closely packed. It shows that the regrowth was initiated from the $\mathrm{GaN}$ seed layer, filled the nanogaps between the silica nanospheres, and continued beyond as a fully coalesced layer, which has been first demonstrated in GaAs selective area deposition. ${ }^{18}$ The lack of polycrystalline nucleation has been confirmed by studying a multilayer sample for which the regrowth was stopped at an early stage. Figure 1c shows the SEM of this sample and reveals that the GaN regrowth takes place rather homogeneously across the surface through the nanogaps in between silica nanospheres but also causes the formation of a number of large crystalline islands (elongated along [0001], with $\{10-11\}$ and (000-1) facets). ${ }^{19}$ Low temperature (15 K) CL image taken at the GaN near band-edge (NBE) emission energy of $3.46 \mathrm{eV}$ (Figure 1d) shows that there is GaN NBE emission from the $3 \mathrm{D}$ islands (as indicated by the white circles) as well as originating from between the nanogaps (red circles). Furthermore, since a BSF is often regarded as a type II zincblende QW layer embedded in a wurtzite matrix, ${ }^{20,21}$ these planar defects strongly luminesce at an energy of $3.40 \mathrm{eV}$. The 
CL image taken at $3.40 \mathrm{eV}$ suggests that the BSFs are only present in the $3 \mathrm{D}$ islands (white circles) but could not propagate through the nanogaps (red circles) as shown in Figure 1e.

By varying the silica nanosphere deposition rate between 0.15 and $0.6 \mathrm{~mm} / \mathrm{min}$, a series of samples were prepared with multilayers and ML with different silica nanosphere coverage, namely densely packed ML and loosely packed ML, as shown in Figure $2 a-c$. Figure $2 d-i$ show the room temperature SEM
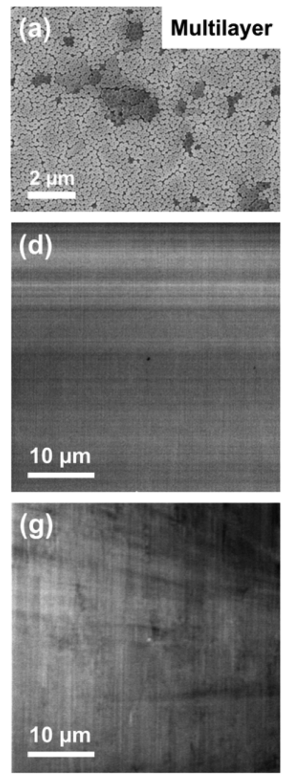
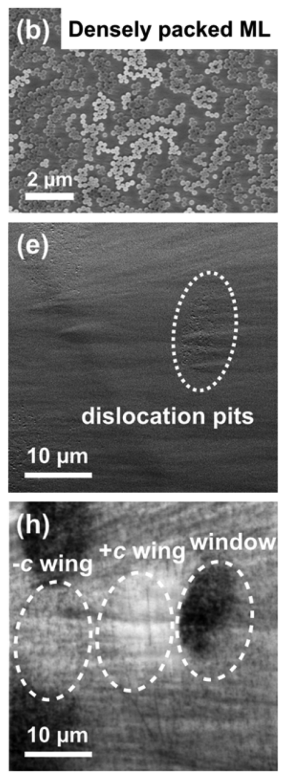

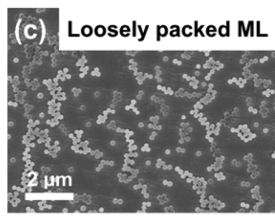

(f)
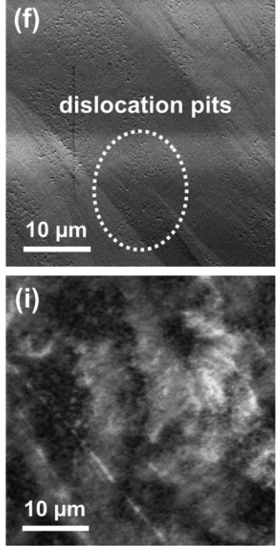

Figure 2. SEM images of silica nanospheres deposited on a GaN/ sapphire template with different deposition rates: (a) $0.15 \mathrm{~mm} / \mathrm{min}$, (b) $0.3 \mathrm{~mm} / \mathrm{min}$, (c) $0.6 \mathrm{~mm} / \mathrm{min}$. Plan-view SEM and roomtemperature panchromatic $\mathrm{CL}$ images of fully coalesced samples with $(\mathrm{d}, \mathrm{g})$ multilayer, $(\mathrm{e}, \mathrm{h})$ densely packed $\mathrm{ML}$, and $(\mathrm{f}, \mathrm{i})$ loosely packed $\mathrm{ML}$ of silica nanospheres.

and cathodoluminescence (CL) data of the fully coalesced samples grown on these nanosphere masked layers. For deposition rates faster than $0.6 \mathrm{~mm} / \mathrm{min}$, a very loosely packed $\mathrm{ML}$ is formed and the CL image of the related overgrown sample is mostly dominated by a very large number of dislocation related dark spots with less than $10 \%$ of the surface appearing bright, which is as expected since most of the dislocations from the seed layer can propagate freely up into the film due to lack of silica nanosphere coverage and thus less blocking by the nanospheres (Sample not shown here: see Supporting Information Figure S2). For the loosely packed ML $(0.6 \mathrm{~mm} / \mathrm{min})$, the silica nanospheres appear in bands of small clusters, and from the overgrown sample, bright regions in the CL map are emerging and have been found to cover $\sim 35 \%$ of the sample area (Figure 2i), implying that some dislocations were blocked during the coalescence process. The location of threading dislocations are visible as small surface depressions in the SEM image in Figure $2 \mathrm{f}$ and as dark spots in the CL image since threading dislocation cores are known to be nonradiative recombination centers. ${ }^{22}$ The CL image of the densely packed ML sample $(0.3 \mathrm{~mm} / \mathrm{min})$ shows a large bright region covering $\sim 52 \%$ of the area with a few narrow bands of dark spots in Figure $2 \mathrm{~h}$. The data show some similarities to samples grown by the conventional ELOG process, a periodic alternation of low defect density and highly defective regions is observed. ${ }^{12,23}$
In particular, there are large areas with a number of dark spots (similar to the $-c$ wing in ELOG), and small areas with almost no dark spots (like the $+c$ wing in ELOG) adjacent to the dislocation clusters (the ELOG window). ${ }^{23}$ In the multilayer sample, however, the bright region has been found to cover over $85 \%$ of the sample area with only a small number of dark spots, indicating a significant reduction of the dislocation density (Figure $2 \mathrm{~g}$ ). A dislocation density of $2 \times 10^{7} \mathrm{~cm}^{-2}$ is estimated for the multilayer sample by counting the dark spots in the CL images (although this may be an underestimation of the dislocation density as two or more dark spots may merge in $\mathrm{CL}$ ), which represents $\sim 4$ orders of magnitude reduction from the $1 \times 10^{11} \mathrm{~cm}^{-2}$ dislocations originally in the seed layer. The optimal number of layers has been found to be $\sim 5$, where not only can the entire surface be covered, but also the nanogaps in between the silica nanospheres will be sufficiently large to allow the regrowth to initiate from the underlying $\mathrm{GaN}$ seed layer. However, the silica nanosphere stack will crack upon drying if the number of layers exceed $\sim 10$, in which case the regrowth only occurs through the cracks (see Supporting Information Figure S3).

Detailed CL analysis of the densely packed ML and the multilayer samples were carried out at $77 \mathrm{~K}$. The $\mathrm{CL}$ spectrum taken from the densely packed ML sample shows emission peaks at $\sim 3.46 \mathrm{eV}$, which can be assigned to the donor bound exciton $\left(\mathrm{D}^{\circ} \mathrm{X}\right)$ in $\mathrm{GaN}$, and at $\sim 3.40 \mathrm{eV}$, which is BSF related emission. On the other hand, the multilayer sample shows a very strong $\mathrm{D}^{\circ} \mathrm{X}$ emission at $\sim 3.46 \mathrm{eV}$ and only a weak BSF emission at $\sim 3.40 \mathrm{eV}$ (Figure 3a). Additionally, the free exciton
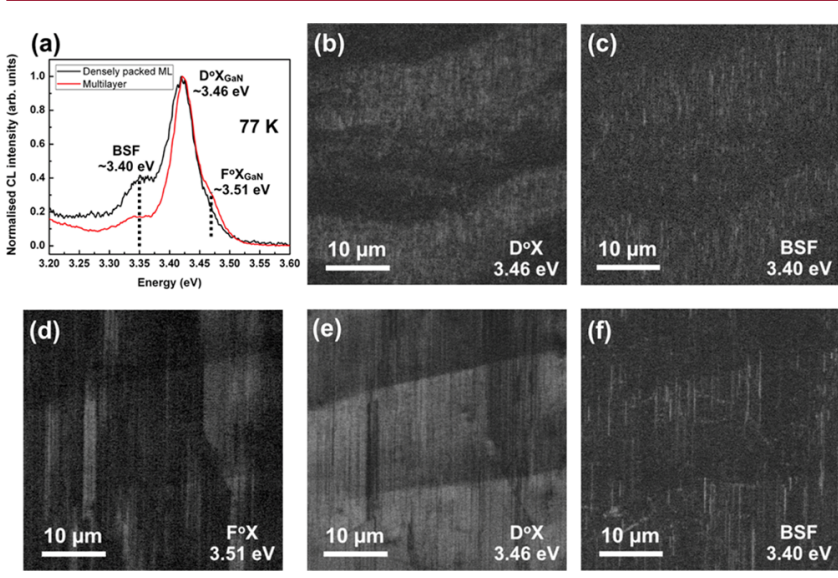

Figure 3. (a) Low temperature ( $77 \mathrm{~K}) \mathrm{CL}$ spectra and monochromatic $C L$ images of ( $b$ and $c$ ) densely packed $M L$ and $(d-f)$ multilayer samples taken at various emission energies corresponding to $\mathrm{F}^{\circ} \mathrm{X}_{\mathrm{GaN}}$ at $\sim 3.51 \mathrm{eV}, \mathrm{D}^{\circ} \mathrm{X}_{\mathrm{GaN}}$ at $\sim 3.46 \mathrm{eV}, \mathrm{BSF}$ emission at $\sim 3.40 \mathrm{eV}$.

peak $\left(\mathrm{F}^{\circ} \mathrm{X}\right)$ observed on the high energy side of the $\mathrm{D}^{\circ} \mathrm{X}$ peak at $\sim 3.51 \mathrm{eV}$ indicates a high optical quality and thus low defect density of the multilayer sample. Note that the small shift $(\sim 7$ $\mathrm{meV}$ ) in the $\mathrm{D}^{\circ} \mathrm{X}$ peak between the two samples can be explained by the presence of different strain states in the samples. ${ }^{24}$ Assessment of the defect reduction efficiency and improvements in the material quality is obtained by evaluating the CL intensity ratio of the GaN bound exciton to the BSF emission. This ratio is found to be 5.8 for the multilayer sample, which is $\sim 2.5$ times larger than that of the densely packed ML sample and $\sim 5$ times larger than that of an ELOG sample $(\sim 1.1)^{25}$ measured under the same conditions in CL (averaged across the ELOG sample over the same area size including both 
the wing and window regions). In addition, CL images taken at different emission energies show that the $\mathrm{GaN} \mathrm{D}^{\circ} \mathrm{X}$ emission is only visible in the bright bands in the densely packed $\mathrm{ML}$ sample (Figure $3 \mathrm{~b}$ ), as the large number of dislocations may have quenched the emission from the other regions. Also, a large number of bright linear features along the [1-100] direction are seen in the BSF emission image (Figure 3c). The multilayer sample shows a uniform $\mathrm{GaN} \mathrm{D}^{\circ} \mathrm{X}$ emission across the CL image shown in Figure 3e, and a much-reduced number of BSF related bright linear features in Figure $3 \mathrm{f}$. An average BSF density of $\sim 3200 \mathrm{~cm}^{-1}$ has been measured for the multilayer sample by measuring the bright linear features. Although this is likely to be an underestimation of the BSF density, as these bright linear features may involve a bundle of BSFs, this provides a lower limit for the BSF density present in the multilayer sample.

Cross-sectional transmission electron microscopy (TEM) was used to further study the microstructure of the multilayer sample and gain insights into the regrowth and defect reduction mechanisms. Figure $4 a$ shows a high-angle annular dark field


Figure 4. Cross-sectional TEM analysis of samples with multilayers of silica nanospheres. (a) HAADF taken at the c-zone $\langle 0001\rangle$ axis and (b) WBDF image taken using $g=11-20$ at close to the $c$-zone $\langle 0001\rangle$ axis, and (c) BF image viewed close to the $a$-zone $\langle 11-20\rangle$ axis, such that the BSFs are visible. (a) and (b) are from the same TEM sample, where the blue arrows point out several dislocations and the yellow ones mark the PSFs. The white arrow marks the position of a coalescence boundary, and the pink arrows indicate the BSFs in (c).

scanning TEM (HAADF-STEM) image taken at the conditions where the direction of the electron beam was parallel to the $\langle 0001\rangle$ zone axis. It reveals the microstructural transitions from the sapphire substrate to the seed GaN layer, presence of voids in the seed layer, to the incorporated multilayers of nanospheres and regrown GaN layer, where a dramatic reduction of defects in the regrown GaN layer can be seen due to the introduction of the MSN. A few dislocations are visible in the regrown GaN layer and are labeled with blue arrows. In order to further analyze the defects, weak beam dark field (WBDF) imaging was also carried out. Figure $4 \mathrm{~b}$ is a WBDF image acquired at $\mathbf{g - g}(\mathbf{g}=11-20)$ conditions close to the $\mathrm{c}$ zone axis from the same sample as in Figure 4a. Under these diffraction conditions, a-type and $(\mathbf{a}+\mathbf{c})$-type dislocations and some partial dislocations are visible, some of which are labeled with blue arrows. In addition, several prismatic stacking faults (PSFs) are also visible, as marked by the yellow arrows. This image confirms that the dislocation density in the $\mathrm{GaN}$ above the MSN is much lower than that in the GaN seed layer, verifying that the dislocations are efficiently blocked by the MSN. There are several underlying dislocation reduction mechanisms that might simultaneously play important roles for achieving such a low dislocation density in the multilayer sample. First, the formation of voids in the seed layer suggests that materials have evaporated through the nanogaps in the MSN during the initial regrowth process, with the exposed surfaces acting to terminate some defects, similar to the $\mathrm{ScN}$ interlayer case. ${ }^{9}$ Second, the dislocations are blocked at the $\mathrm{GaN} /$ nanosphere interface, similar to dislocation blocking at an ELOG mask. A third mechanism is the nucleation and epitaxial growth of $\mathrm{GaN}$ through the channels in the MSN (as shown earlier in Figure 1c) and the subsequent lateral coalescence process. Dislocation annihilation resulting from island coalescence is evidenced by the formation of half-loops between bent-over dislocations during lateral growth, as marked by the blue arrow with an asterisk in Figure $4 a$ and $4 b$.

Figure $4 \mathrm{c}$ shows a bright field (BF) TEM image taken from another sample, in which the sample was oriented at close to the $\langle 11-20\rangle$ zone axis so that the BSFs are in contrast. The BSFs in the regrown GaN layer are observed as narrow dark bands extending toward the sample surface some of which are labeled with pink arrows. In contrast, in the GaN seed layer, it is almost impossible to distinguish individual BSFs in the GaN seed layer since their density is so high. A closer look at the $\mathrm{GaN} /$ silica nanosphere interface reveals that the majority of the BSFs have been blocked by the MSN, but new BSFs appear to have been initiated at the interfaces between the silica nanosphere and the regrown $\mathrm{GaN}$ layers, possibly being formed during island coalescence. ${ }^{26}$ The BSF density above the silica nanospheres can be estimated using these TEM images, giving a value of $\sim 3 \times 10^{5} \mathrm{~cm}^{-1}$, which is a higher value than the BSF densities measured using the CL data. It should be noted that the estimation of the BSF density in this case assumes that all BSFs run across the entire thickness of the
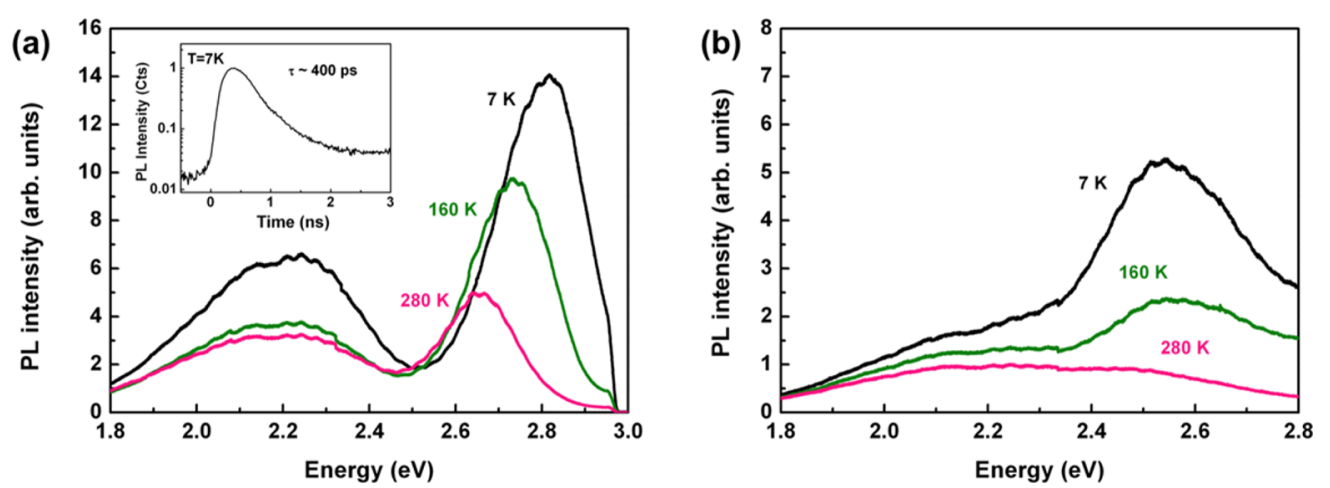

Figure 5. Temperature dependent photoluminescence (PL) spectra taken from samples (a) with and (b) without the integration of MSN in the $\mathrm{GaN}$ template (essentially a GaN seed layer). Inset shows a PL decay spectrum taken at $7 \mathrm{~K}$, revealing a short exciton lifetime $\sim 400$ ps. 
TEM foil, so this value represents an upper bound on the BSF density. The differences in the TEM and CL data can be further explained by the limited size of the TEM specimen. Overall, these data show that the use of a self-assembled MSN can dramatically reduce the dislocation density in nonpolar $a$-plane $\mathrm{GaN}$ with a simple one-step regrowth process and can also reduce the BSF density by one or 2 orders of magnitude compared to the seed layer which has a BSF density $\sim 1 \times 10^{6}$ $\mathrm{cm}^{-1}$.

To assess the impact of the change in defect densities on the optical efficiency of QW overgrown layers, 5 periods of InGaN/ $\mathrm{GaN}$ MQWs were grown with an In fraction $(\mathrm{x})$ of 0.15 in $\mathrm{In}_{x} \mathrm{Ga}_{1-x} \mathrm{~N}$, and QW and barrier thicknesses of $4 \pm 0.2 \mathrm{~nm}$ and $7 \pm 0.2 \mathrm{~nm}$ on samples with and without the MSN (essentially a GaN seed layer). Temperature dependent PL spectra of the samples with MSN and without MSN are shown in Figure 5, where the $\mathrm{QW}$ emission peak at $7 \mathrm{~K}$ is centered at $\sim 2.8 \mathrm{eV}$ and $\sim 2.6 \mathrm{eV}$, respectively. It is known that the use of silica nanospheres can result in less strained GaN layers. ${ }^{27}$ Although the two samples were grown at the same time, the sample without the MSN may exhibit much greater wafer bowing and inhomogeneity in the InGaN growth temperature and thus give rise to a different QW emission energy. In addition, the differences in the nanoscale surface morphology and indeed local differences in indium incorporation at dislocation and stacking faults will have a greater effect on the QW emission energy in the sample without the MSN. In both samples, a weaker and broader emission band at $\sim 2.2 \mathrm{eV}$ is present and is attributed to impurity-related recombination (on the basis of the very long PL decay times $(>10 \mu \mathrm{s})$. At $7 \mathrm{~K}$, the PL decay time of the QW emission is around $\sim 400$ ps (see inset of Figure 5a), consistent with the absence of built-in electric fields across the QW. ${ }^{28}$ The monotonic temperature dependence of the QW PL peak energy shift and its magnitude $(\sim 160 \mathrm{meV})$ is also consistent with other reports on the optical properties of nonpolar InGaN QWs. ${ }^{28-30}$ Due to the complex interplay between the BSF emission and the true QW emission in the temperature dependent PL data, ${ }^{3}$ it is not possible to analyze the data to extract meaningful internal quantum efficiencies. Nonetheless, it is notable that the ratio of the integrated QW PL intensity of the MSN sample between $7 \mathrm{~K}$ and room temperature is more than 33 times larger than that of the sample without the MSN. We attribute this large enhancement to the greatly reduced defect densities in the GaN template prepared by the MSN method. To our knowledge, this marks by far the largest enhancement in the emission efficiency of nonpolar a-plane InGaN/GaN MQWs when compared to ELOG or other patterning techniques. ${ }^{14,31}$ Furthermore, even at $7 \mathrm{~K}$, the PL intensity of the QW emission (and impurity emission) in the sample with MSNs is markedly greater (by a factor of $\sim 5$ ) than it is for the sample with no MSNs. This is likely to be due to enhanced light extraction efficiency related to the refractive index contract between the GaN and the silica nanospheres, i.e. the MSN acts as a reflector. ${ }^{14}$

2.2. Semipolar (11-22) GaN. To demonstrate the applicability of the MSN technique to GaN grown on other orientations, it has also been applied to reduce the defect density in semipolar (11-22) GaN grown on $m$-plane sapphire in MOCVD. A cross-sectional SEM image of an uncoalesced semipolar multilayer sample and a schematic representation of the regrowth process are shown in Figure $6 a$ and $6 \mathrm{~b}$, respectively. The growth conditions were chosen to achieve an enhanced growth rate along the [0001] direction. GaN
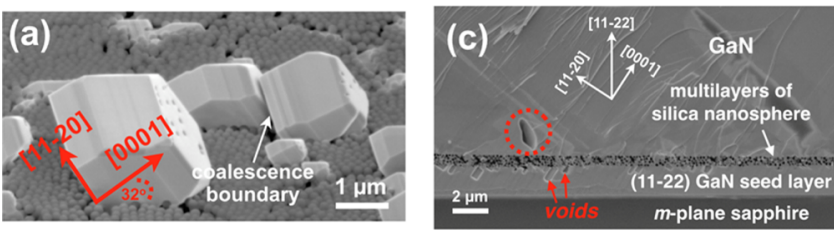

(b)
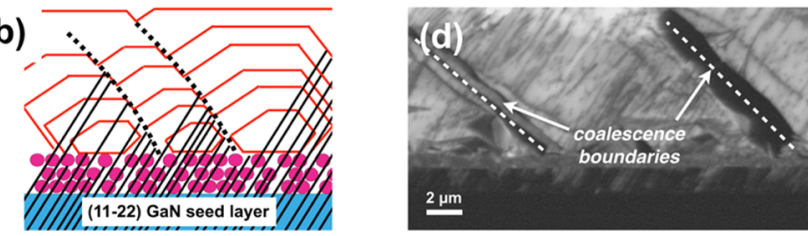

Figure 6. (a) SEM image of the semipolar (11-22) GaN regrowth at an early stage and (b) schematic illustration of the crystal growth and defect reduction process (dark lines represents the defects, and dotted lines indicate the position of the coalescence boundaries). Room temperature cross-sectional (c) SEM and (d) CL images of the fully coalesced multilayer sample, where a coalescence void (red dotted circle) and the coalescence boundaries (white dashed lines) can be seen.

grows through the gaps between the silica nanospheres, and then islands grow along the inclined $c$-axis $\left(\sim 32^{\circ}\right.$ to the film surface) and coalesce. At the resulting voids or coalescence boundaries, both dislocations and BSFs may be completely blocked due to the asymmetric growth profile. Therefore, in the case of incorporating multilayers of silica nanospheres in the growth of semipolar (11-22) GaN, the growth and defect reduction mechanisms appear to be similar to the asymmetric ELOG process. ${ }^{16,32}$ Figure $6 \mathrm{c}$ shows a cross-sectional SEM image of the fully coalesced multilayer sample, which reveals the presence of a coalescence void above the silica nanospheres (red dotted circle). As in the nonpolar multilayer sample, there are also voids formed in the seed layer during the initial regrowth process, which were not observed for the ML samples (See Supporting Information Figure S4). Coalescence boundaries can be seen in the CL image in Figure 6d, which have been marked by the white dashed line.

Similar to the nonpolar $\mathrm{GaN}$, the room temperature CL images of the ML samples are mostly dominated by a very large number of dislocation-associated dark spots (See Supporting Information Figure S5). Figure $7 \mathrm{a}$ and $\mathrm{b}$ shows the room temperature SEM and CL images of the multilayer sample. The multilayer sample exhibits very large bright areas with many fewer dark spots, suggesting that the MSN has reduced the dislocation density significantly. The dislocation density in the multilayer sample has been measured to be $\sim 3 \times 10^{8} \mathrm{~cm}^{-2}$ by counting the dark spots observed in the CL images. Note that there are some chevron-shaped surface features pointing toward [11-23] present in all samples as shown in Figure $7 \mathrm{a}$, which are also evident in the corresponding CL image in Figure $7 \mathrm{~b}$. These are commonly observed in (11-22) $\mathrm{GaN}$ and have been attributed to asymmetric growth fronts and the subsequent coalescence process. ${ }^{16,33}$ It is worth noting that the presence of dislocation clusters might imply that additional dislocations were generated to compensate the misorientation between growth facets during the coalescence process. ${ }^{34}$

Low temperature $(77 \mathrm{~K}) \mathrm{CL}$ spectra of the multilayer and the densely packed ML samples are shown in Figure 7c. Both samples exhibit very strong luminescence from the $\mathrm{GaN} \mathrm{D}^{\circ} \mathrm{X}$ recombination at $\sim 3.46 \mathrm{eV}$, relatively weak emission from the BSFs at $\sim 3.37 \mathrm{eV}$, and negligible PSF emission at around 3.28 

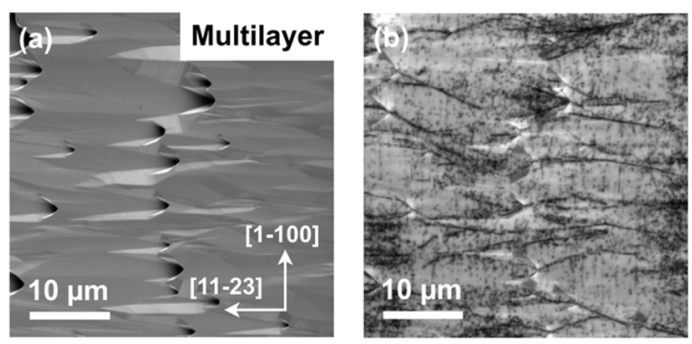

(c)



Figure 7. Plan-view room temperature (a) SEM and (b) CL images of fully coalesced multilayer sample. (c) Low temperature CL spectra taken from both multilayer (red) and densely packed ML samples (black). (d) CL image taken at the BSF emission energy at $3.37 \mathrm{eV}$ from the multilayer sample.

$\mathrm{eV}$. BSF emission features can be seen as bright linear features along the $[1-100]$ direction in the monochromatic CL image (Figure $7 \mathrm{~d}$ ). A BSF density of $\sim 2400 \mathrm{~cm}^{-1}$ has been estimated and determined by the CL images. And the CL intensity ratio between the $\mathrm{D}^{\circ} \mathrm{X}$ and the $\mathrm{BSF}$ emission has been found to be $\sim 9.6$ for the multilayer sample, which is more than two times larger than that of an asymmetric semipolar ELOG sample $(\sim 4.3)^{16}$ measured under the same conditions in CL (averaged across the ELOG sample over the same area size including both the wing and window regions). Other samples with MLs of silica nanospheres have also been explored (as in the former nonpolar case), but the MSN gives the best results for both dislocation and BSF reduction. Given that defect reduction is achieved via island nucleation through the nanogaps between the silica nanospheres in a self-assembled fashion, a small number of BSFs can still propagate up to the top surface due to local nonuniformity in the initial island growth and the subsequent coalescence and defect blocking process. Further optimization of the MSN deposition and growth conditions is thus needed to achieve BSF-free (11-22) semipolar GaN.

\section{CONCLUSION}

Epitaxial integration of self-assembled multilayers of silica nanospheres in heteroepitaxial growth of non- and semipolar $\mathrm{GaN}$ is demonstrated as a new defect reduction technique, which not only reduces the dislocations down to $10^{7} \mathrm{~cm}^{-2}$ and $\sim 3 \times 10^{8} \mathrm{~cm}^{-2}$, respectively, but also allows us to significantly reduce the BSF density by more than an order of magnitude in both cases. The strong luminescence enhancement in nonpolar InGaN/GaN MQWs grown on the template prepared by the MSN technique is believed to relate to both improved internal quantum efficiency and enhanced light extraction, demonstrating that this is a successful multifunctional approach to improving optical performance. The MSN technique could be beneficial for heteroepitaxial growth of other lattice-mismatched materials, such as GaN-on-silicon and cubic GaN.

\section{EXPERIMENTAL SECTION}

The non- and semipolar GaN samples were grown by MOCVD in a 6 $\times 2$ in. Thomas Swan close-coupled showerhead reactor on $r$-plane $(1-102)$ and $m$-plane $(1-100)$ sapphire substrates, respectively. Trimethylgallium, trimethylindium, and ammonia were used as precursors. Hydrogen and nitrogen were used as carrier gas for $\mathrm{GaN}$ and InGaN/GaN QWs, respectively. Nonpolar (11-20) GaN seed layer of $\sim 1 \mu \mathrm{m}$ was first grown with a V/III ratio of $\sim 60$ at $1050{ }^{\circ} \mathrm{C}$, following a $30 \mathrm{~nm} \mathrm{GaN}$ nucleation layer grown at $540{ }^{\circ} \mathrm{C}$ and 500 Torr. This $a$-plane GaN seed layer ${ }^{26}$ typically has a dislocation density of $2 \times 10^{11} \mathrm{~cm}^{-2}$, and a BSF density of $1 \times 10^{6} \mathrm{~cm}^{-1}$. Semipolar (1122) $\mathrm{GaN}$ seed layer of $\sim 2 \mu \mathrm{m}$ was grown under similar growth conditions as the nonpolar seed layer, but with a V/III ratio of $\sim 800$ and has a dislocation and BSF density of $3.0 \times 10^{10} \mathrm{~cm}^{-2}$ and $3.2 \times$ $10^{5} \mathrm{~cm}^{-1}$, respectively. ${ }^{9}$

Then the wafers were followed by an oxygen plasma treatment for 5 min to make the GaN surface hydrophilic. Silica nanospheres ( $d=$ $\sim 180 \mathrm{~nm}$, homemade ${ }^{35}$ ) were dispersed in ethanol and the nanosphere suspension contained $0.6 \% \mathrm{v} / \mathrm{v}$ of silica. Coating on the $\mathrm{GaN}$ seed layer was performed by injecting $0.2 \mathrm{~mL}$ of suspension at the edge of an inclined, precleaned glass slide $(25 \times 75 \mathrm{~mm})$ and pulling the slide at a constant angle to the surface away from the meniscus using a syringe pump as a linear motor. At room temperature, the following speeds resulted in $0.15 \mathrm{~mm} / \mathrm{min}-$ multilayer, (b) $0.3 \mathrm{~mm} / \mathrm{min}$-dense, but less than close-packed monolayer coverage, and (c) $0.6 \mathrm{~mm} / \mathrm{min}$-loose monolayer coverage. Regrowth was initially carried out with a V/III ratio of 60 at $1050{ }^{\circ} \mathrm{C}$ and 100 Torr to promote lateral growth and completed by the growth of another $2 \mu \mathrm{m}$ of $\mathrm{GaN}$ with a $\mathrm{V} / \mathrm{III}$ ratio of 740 to improve luminescence properties. $^{23}$

SEM-CL studies were performed on a liquid helium cooled stage in a Philips XL30s SEM operating at $5 \mathrm{kV}$ and equipped with a Gatan MonoCL4 system. A dual beam focused ion beam (FIB) microscope (FEI Helios NanoLab) was used to prepare TEM lamellas whose surface normals are close to the $c$-zone $\langle 0001\rangle$ and $m$-zone $\langle 1-100\rangle$ axis respectively, according to the standard in situ lift-out technique. Figure $1 \mathrm{~b}$ is a cross-sectional image of the sample which was taking before being lift-out. Two FEI TEM microscopes (FEI Tecnai Osiris and F20) with field-emission guns were used to investigate the samples at operating voltage $200 \mathrm{kV}$ using bright-field and dark-field imaging modes and HAADF-STEM mode where applicable. In order to bring BSF in contrast, the TEM lamella with the $m$-zone $\langle 1-100\rangle$ normal was tilted to be approximately adjacent to the a-zone $\langle 11-20\rangle$ axis. $^{5}$

PL was excited using pulsed excitation from a $3.147 \mathrm{eV}$ diode laser, using an average excitation density of $\sim 100 \mathrm{~W} / \mathrm{cm}^{2}$. The emission was collected and focused into a spectrograph equipped with a UV enhanced CCD array. The sample temperature was controlled using a He-flow microstat. To record the PL transients, the spectrally dispersed signal was detected using a photomultiplier tube and processed using time-correlated single photon counting.

\section{ASSOCIATED CONTENT}

\section{S Supporting Information}

The Supporting Information is available free of charge on the ACS Publications website at DOI: 10.1021/acs.cgd.5b01560Datasets for the figures in this paper can be found at https:// www.repository.cam.ac.uk/handle/1810/253190.

Cross-sectional SEM images of fully coalesced nonpolar a-plane samples; images of a very loosely packed monolayer silica nanosphere sample; images of an uncoalesced multilayer sample; images of a fully coalesced semipolar (11-22) sample with a densely packed ML of silica nanospheres; and images of fully coalesced semipolar (11-22) samples (PDF) 


\section{AUTHOR INFORMATION}

\section{Corresponding Authors}

*E-mail: tz234@cam.ac.uk (TZ).

*E-mail: sks46@cam.ac.uk (SKS).

Notes

The authors declare no competing financial interest.

\section{ACKNOWLEDGMENTS}

This work has been funded in part by the EPSRC (Grant No. $\mathrm{EP} / \mathrm{J} 003603 / 1$ and EP/M011682/1) and by the European Research Council under the European Community's Seventh Framework Programme (FP7/2007-2013)/ERC grants agreement no 279361 (MACONS) and no 280078 (EMATTER).

\section{REFERENCES}

(1) Verzellesi, G.; Saguatti, D.; Meneghini, M.; Bertazzi, F.; Goano, M.; Meneghesso, G.; Zanoni, E. J. Appl. Phys. 2013, 114, 071101.

(2) Brinkley, S. E.; Lin, Y.-D.; Chakraborty, A.; Pfaff, N.; Cohen, D.; Speck, J. S.; Nakamura, S.; DenBaars, S. P. Appl. Phys. Lett. 2011, 98, 011110.

(3) Kundys, D.; Schulz, S.; Oehler, F.; Sutherland, D.; Badcock, T. J.; Dawson, P.; Kappers, M. J.; Oliver, R. A.; Humphreys, C. J. J. Appl. Phys. 2014, 115, 113106.

(4) Ko, Y.-H.; Kim, J.-H.; Jin, L.-H.; Ko, S.-M.; Kwon, B.-J.; Kim, J.;

Kim, T.; Cho, Y.-H. Adv. Mater. 2011, 23, 5364-5369.

(5) Johnston, C. F.; Kappers, M. J.; Moram, M. A.; Hollander, J. L.; Humphreys, C. J. Phys. Status Solidi A 2009, 206, 1190-1193.

(6) Lee, S.-N.; Kim, J.; Kim, H. J. Cryst. Growth 2011, 326, 19-22.

(7) Sun, Q.; Kong, B. H.; Yerino, C. D.; Ko, T.-S.; Cho, H. K.; Han, J. J. Appl. Phys. 2009, 106, 123519.

(8) Oehler, F.; Sutherland, D.; Zhu, T.; Emery, R.; Badcock, T. J.; Kappers, M. J.; Humphreys, C. J.; Dawson, P.; Oliver, R. A. J. Cryst. Growth 2014, 408, 32-41.

(9) Johnston, C. F.; Moram, M. A.; Kappers, M. J.; Humphreys, C. J. Appl. Phys. Lett. 2009, 94, 161109.

(10) Araki, M.; Hoshino, K.; Tadatomo, K. Phys. Status Solidi C 2007, 4, 2540-2543.

(11) Wieneke, M.; Noltemeyer, M.; Bastek, B.; Rohrbeck, A.; Witte, H.; Veit, P.; Blasing, J.; Dadgar, A.; Christen, J.; Krost, A. Phys. Status Solidi B 2011, 248, 578-582.

(12) Vennéguès, P. Semicond. Sci. Technol. 2012, 27, 024004.

(13) Imer, B. M.; Wu, F.; DenBaars, S. P.; Speck, J. S. Appl. Phys. Lett. 2006, 88, 061908.

(14) Park, S. H.; Park, J.; You, D.-J.; Joo, K.; Moon, D.; Jang, J.; Kim, D.-U.; Chang, H.; Moon, S.; Song, Y.-K.; Lee, G.-D.; Jeon, H.; Xu, J.; Nanishi, Y.; Yoon, E. Appl. Phys. Lett. 2012, 100, 191116.

(15) Prevo, B. G.; Velev, O. D. Langmuir 2004, 20, 2099-2107.

(16) Zhu, T.; Sutherland, D.; Badcock, T. J.; Hao, R.; Moram, M. A.; Dawson, P.; Kappers, M. J.; Oliver, R. A. Jpn. J. Appl. Phys. 2013, 52, 08JB01.

(17) Haskell, B. A.; Nakamura, S.; DenBaars, S. P.; Speck, J. S. Phys. Status Solidi B 2007, 244, 2847-2858.

(18) Nelson, E. C.; Dias, N. L.; Bassett, K. P.; Dunham, S. N.; Verma, V.; Miyake, M.; Wiltzius, P.; Rogers, J. A.; Coleman, J. J.; Li, X.; Braun, P. V. Nat. Mater. 2011, 10, 676-681.

(19) Sun, Q.; Yerino, C. D.; Ko, T. S.; Cho, Y. S.; Lee, I.-H.; Han, J.; Coltrin, M. E. J. Appl. Phys. 2008, 104, 093523.

(20) Rebane, Y. T.; Shreter, Y. G.; Albrecht, M. Phys. Status Solidi A 1997, 164, 141-144.

(21) Liu, R.; Bell, A.; Ponce, F. A.; Chen, C. Q.; Yang, J. W.; Khan, M. A. Appl. Phys. Lett. 2005, 86, 021908.

(22) Sugahara, T.; Sato, H.; Hao, M.; Naoi, Y.; Kurai, S.; Tottori, S.; Yamashita, K.; Nishino, K.; Romano, L.; Sakai, S. Jpn. J. Appl. Phys. 1998, 37, L398-L400.

(23) Häberlen, M.; Badcock, T. J.; Moram, M. A.; Hollander, J. L.; Kappers, M. J.; Dawson, P.; Humphreys, C. J.; Oliver, R. A. J. Appl. Phys. 2010, 108, 033523.
(24) Gil, B.; Briot, O.; Aulombard, R. L. Phys. Rev. B: Condens. Matter Mater. Phys. 1995, 52, R17028-R17031.

(25) Sutherland, D.; Oehler, F.; Zhu, T.; Griffiths, J. T.; Badcock, T. J.; Dawson, P.; Emery, R. M.; Kappers, M. J.; Humphreys, C. J.; Oliver, R. A. Phys. Status Solidi C 2014, 11, 541-544.

(26) Hao, R.; Kappers, M. J.; Moram, M. A.; Humphreys, C. J. J. Cryst. Growth 2011, 337, 81-86.

(27) Kim, J.; Woo, H.; Joo, K.; Tae, S.; Park, J.; Moon, D.; Park, S. H.; Jang, J.; Cho, Y.; Park, J.; Yuh, H.; Lee, G.-D.; Choi, I.-S.; Nanishi, Y.; Han, H. N.; Char, K.; Yoon, E. Sci. Rep. 2013, 3, 3201.

(28) Chiu, C. H.; Kuo, S. Y.; Lo, M. H.; Ke, C. C.; Wang, T. C.; Lee, Y. T.; Kuo, H. C.; Lu, T. C.; Wang, S. C. J. Appl. Phys. 2009, 105, 063105.

(29) Roberts, A. T.; Mohanta, A.; Everitt, H. O.; Leach, J. H.; Van Den Broeck, D.; Hosalli, A. M.; Paskova, T.; Bedair, S. M. Appl. Phys. Lett. 2013, 103, 181106.

(30) Langer, T.; Pietscher, H.-G.; Ketzer, F. A.; Jönen, H.; Bremers, H.; Rossow, U.; Menzel, D.; Hangleiter, A. Phys. Rev. B: Condens. Matter Mater. Phys. 2014, 90, 205302.

(31) Son, J.-S.; Honda, Y.; Yamaguchi, M.; Amano, H. Jpn. J. Appl. Phys. 2014, 53, 05FL01.

(32) de Mierry, P.; Kriouche, N.; Nemoz, M.; Nataf, G. Appl. Phys. Lett. 2009, 94, 191903.

(33) Caliebe, M.; Han, Y.; Hocker, M.; Meisch, T.; Humphreys, C. J.; Thonke, K.; Scholz, F. Phys. Status Solidi B 2015, DOI: 10.1002/ pssb.201552266.

(34) Kriouche, N.; Vennéguès, P.; Nemoz, M.; Nataf, G.; de Mierry, P. J. Cryst. Growth 2010, 312, 2625-2630.

(35) Liu, Z.-F.; Ding, T.; Zhang, G.; Song, K.; Clays, K.; Tung, C.-H. Langmuir 2008, 24, 10519-10523. 